\title{
Reproductive mental health risk in Nigeria: myths, facts and challenges
}

\author{
Abiodun 0. Adewuya ${ }^{1}$ and Olutayo O. Aloba ${ }^{2}$
}

'Department of Behavioural Medicine, Lagos State University College of Medicine, 1-3, Oba Akinjobi Way, Ikeja, PMB 21266, Lagos, Nigeria, email biodunwuya@yahoo.com

${ }^{2}$ Department of Mental Health, Obafemi Awolowo University Teaching Hospital, Ile-Ife, Nigeria

\begin{abstract}
$\mathrm{t}$ is widely known that Africans and especially Nigerians place much emphasis on childbearing. It has been said that the effect of childbirth relates to the society and culture's response to parenthood and the existing family structure. Many rituals exist in African societies to signify the changes in women's identity, roles and status during pregnancy and following childbirth. Earlier studies have suggested that perinatal emotional distress is rare among women in sub-Saharan Africa, with the supposed intact family structure in the region acting as a protective factor.

But the fact on ground is that urbanisation has eroded the seemingly intact family structure in Nigeria and the previously perceived low rates of perinatal emotional problems are actually due to the dearth of studies in the region. Also, the instruments and scales used to diagnose emotional problems may not be appropriate to sub-Saharan African peoples, who are known to somatise their emotional problems.
\end{abstract}

\section{Pregnancy}

Although the majority of Nigerian women enjoy a happy and healthy pregnancy and are considered to 'bloom' during this period, studies have shown that pregnancy does not protect them from depression and anxiety. On the contrary, there are increases in psychiatric morbidity during pregnancy. In a study of 180 Yoruba women in south-western Nigeria in late pregnancy, Adewuya et al (2006a) found that $8.3 \%$ met the DSM-IV criteria for current (2 weeks) depressive disorder. The factors independently associated with depression included being single, divorced/separated, polygamous, having a history of stillbirth and perceived lack of social support.

In another study in Nigeria, the rate of anxiety disorders (again meeting DSM-IV criteria) was found to be $39.0 \%$ among women in late pregnancy. This was significantly higher than in the non-pregnant population. The correlates of anxiety disorders included younger age, primiparity and the presence of medical problems (Adewuya et al, 2006b).

\section{Postpartum problems}

\section{Maternity blues}

Maternity blues, or 'baby blues', are transitory mood changes that may begin within the first few days after delivery and last from 1 day through the first 10 days postpartum or longer. Questions have been raised about the cross-cultural validity of this phenomenon but a study of 502 postpartum women with normal delivery in Nigeria (Adewuya, 2005) revealed a rate of $31.3 \%$, with the predictors of maternity blues being significant mood changes in pregnancy, admission during the pregnancy, female baby and single motherhood. Also, maternity blues and mood changes in the early postnatal period have been found to be strong predictors of postnatal depression in Nigerian women (Adewuya, 2006).

\section{Postnatal depression}

Postnatal depression (PND) is the most common mood disorder associated with childbirth. Apart from inflicting profound psychological suffering on new mothers, PND affects marital relationships and adversely affects the emotional and cognitive development of the infant.

The prevalence of PND does not seem to vary across cultures. Using the non-patient version of the Structured Clinical Interview for DSM-III-R (SCID-NP) to assess 876 newly delivered mothers at 6 weeks postpartum, Adewuya et al (2005) found a rate of $14.6 \%$, which is comparable with that obtained in other cultures. The correlates of PND included hospital admission during pregnancy, female gender of the baby, preterm delivery, instrumental delivery, Caesarean section and single motherhood.

\section{Postnatal anxiety}

Few studies have examined anxiety symptoms in the postpartum period, and still fewer in sub-Saharan Africa. This may be because postnatal anxiety is often assumed to be part of postnatal depression. However, anxiety disorders have differing symptoms and aetiological factors that require different approaches, identification, management and preventive strategies. Using the Zung Self-Rating Anxiety and Depression Scales in a repeated cross-sectional study of postpartum women, Adewuya \& Afolabi (2005) found both anxiety and depressive symptoms more frequently in the first 3 weeks postpartum than later. Anxiety was more common than depression in the first 4 weeks, with reversal of the trend subsequently, though with both types of symptom persisting through to the late postpartum period.

\section{Postnatal post-traumatic stress disorder}

Several authors have proposed that post-traumatic stress disorder (PTSD) may occur after a distressing labour or delivery; that is, a difficult or traumatic birth may act as a significant stressor and living through the experience might trigger the symptoms of PTSD. Obstetric practice is generally poor in Africa. There are high rates of delivery at home, in mission houses and with the traditional birth attendants, 
and these are associated with higher maternal mortality and morbidity. In an assessment of 876 women at 6 weeks postpartum, Adewuya et al (2006c) found a prevalence rate of $5.9 \%$ for PTSD after traumatic childbirth, with the correlates including hospital admission due to pregnancy complications, instrumental deliveries and poor maternal control during childbirth.

\section{Effect on infants}

Poor infant growth and under-nutrition are prevalent in subSaharan Africa, especially in Nigeria. Adewuya et al (2008) examined the contribution of maternal depression to this in a longitudinal case-control study and found that infants of mothers with depression had significantly poorer growth than infants of non-depressed mothers after 3 and 6 months. Mothers who are depressed are likely to stop breastfeeding earlier and their infants are more likely to have episodes of diarrhoea and infectious illnesses.

\section{Screening}

In Nigeria medical personnel are scarce, but the identification of depression in pregnant women and newly delivered mothers could be improved by systematic screening in primary care centres, using self-report questionnaires. The Edinburgh Postnatal Depression Scale (EPDS) is intended for this purpose and has been found useful in both the antenatal (Adewuya et al, 2006d) and the postnatal period (Adewuya et al, 2005).

\section{Aetiological factors}

There are several explanations for this seemingly higher reproductive mental health risk among Nigerian women. A review of the literature revealed that the possible correlates and associated factors could be grouped into three categories: obstetric practice factors, baby factors and family factors.

\section{Obstetrics practice factors}

Obstetric practice is still very poor in Nigeria. Most women do not use any health services when pregnant, which makes detection of health problems in pregnancy difficult; there is a high rate of delivery outside hospital. In hospital, most of the operative deliveries are unplanned and mainly done for unbooked patients who have complications either in pregnancy or during delivery. Most Nigerian women want to deliver by themselves because of the pride associated with unassisted delivery in Nigeria and the loss of self-esteem associated with mothers who have operative deliveries (Loto et al, 2009). Furthermore, operative procedures in the country are still associated with high rates of maternal sepsis, morbidity and mortality.

\section{Baby factors}

Nigerians have a deep-rooted preference for male children. In Nigeria, women are blamed for the gender of the baby, so that giving birth to a girl, especially if the woman is delivering for the first time or has not had a male child, could threaten her mental health. Lack of a male child is a frequent cause of marital break-up or of the husband marrying another wife.

Another baby factor is preterm delivery or low birth weight. Poor maternal nutrition during pregnancy and prematurity may result in low birth weight. There are few neonatal intensive-care units in Nigeria and so a much reduced chance of survival for preterm and low birth weight babies, and most mothers have a fatalistic attitude towards ill or preterm neonates.

\section{Family factors}

Topmost on the list of family factors is single motherhood. This may suggest an association between depressive symptoms and a lack of intimacy or support from partners. In a traditional Nigerian setting, any woman who becomes pregnant while not having a husband is viewed as promiscuous, and single parenting is socially unacceptable. The stigma associated with this may contribute to depressed mood.

Another family factor is polygamy, which is practised widely in Nigeria and is a potential source of marital disharmony. Women from polygamous homes tend to receive less spousal support than their counterparts from monogamous homes. In most polygamous marriages, the new mother (who is busy attending to her baby) is often left alone by the father, who seeks sexual gratification with his other wives.

\section{Conclusion}

The primary thing we need is more education regarding maternal and child health in the perinatal period. There should also be a cultural reorientation regarding the family and the gender of the baby. Maternal and infant health policies, a priority in low-income countries, should integrate perinatal mental health as an issue of public importance. Interventions should target mothers in both the antenatal and the postnatal periods. When planning healthcare services or formulating a predictive model for perinatal emotional distress, attention needs to be paid to socio-demographic and obstetric risk factors that are specific to sub-Saharan Africa.

Screening is essential. Routine screening using the EPDS or other instruments should be incorporated into perinatal health services. Prompt referral and treatment of identified cases should be institutionalised.

\section{References}

Adewuya, A. O. (2005) The maternity blues in western Nigerian women: prevalence and risk factors. American Journal of Obstetrics and Gynaecology, 193, 1522-1525.

Adewuya, A. O. (2006) Early postpartum mood as a risk factor for postnatal depression in Nigerian women. American Journal of Psychiatry, $163,1435-1437$.

Adewuya, A. O. \& Afolabi, O. T. (2005) The course of anxiety and depressive symptoms in Nigerian postpartum women. Archives of Women's Mental Health, 8, 257-259.

Adewuya, A. O., Eegunranti, B. A. \& Lawal, A. M. (2005) Prevalence of postnatal depression in western Nigerian women: a controlled study. International Journal of Psychiatry in Clinical Practice, 9, 60-64.

Adewuya, A. O., Ola, B. A., Aloba, O. O., et al (2006a) Prevalence and correlates of depression in late pregnancy among Nigerian women. Depression and Anxiety, 14, 15-21. 
Adewuya, A. O., Ola, B. A., Aloba, O. O., et al (2006b) Anxiety disorders among Nigerian women in late pregnancy: a controlled study. Archives of Women's Mental Health, 9, 325-328.

Adewuya, A. O., Ologun, Y. A. \& Ibigbami, O. S. (2006c) Post-traumatic stress disorder after childbirth in Nigerian women: prevalence and risk factors. British Journal of Obstetrics and Gynaecology, 113, 284-288.

Adewuya, A. O., Ola, B. A., Dada, A. O., et al (2006d) Validation of the Postnatal Depression Scale as a screening tool for depression in late pregnancy among Nigerian women. Journal of Psychosomatic Obstetrics and Gynecology, 27, 267-272.

Adewuya, A. O., Ola, B. A., Aloba, O. O., et al (2008) Impact of postnatal depression on infants' growth in Nigeria. Journal of Affective Disorders, 108, 191-193.

Loto, O. M., Adewuya, A. O., Ajenifuja, O. K., et al (2009) The effect of caesarean section on self-esteem amongst primiparous women in south-western Nigeria: a case control study. Journal of Maternal-Fetal and Neonatal Medicine, 1-5 (epub ahead of print).

\title{
The Stree Arogya Shodh: investigating gynaecological morbidities and women's mental health in India
}

\author{
Vikram Patel
}

Professor of International Mental Health and Wellcome Trust Senior Clinical Research Fellow in Tropical Medicine, London School of Hygiene and Tropical Medicine and Sangath, Goa, India, email vikram.patel@lshtm.ac.uk

\begin{abstract}
G ynaecological complaints have long been associated with poor mental health, in particular depression and anxiety, in higher-income countries. Lower abdominal pain (both menstrual and non-menstrual) and pelvic pain are considered the hallmark gynaecological symptoms associated with somatoform, stress-related and neurotic disorders (the 'common mental disorders', CMDs). Cultural and social factors heavily influence the expression of somatic symptoms and metaphors for emotional distress, but little is known about gynaecological psychosomatic clinical syndromes in non-Western cultures, despite the enormous global health significance of gynaecological symptoms. This paper considers the specific example of the syndrome of abnormal vaginal discharge (AVD) in India.
\end{abstract}

\section{Abnormal vaginal discharge}

Community studies in India show that about half of all women have at least one current gynaecological complaint, of which AVD is the most common. It is characterised by the complaint of vaginal discharge being 'abnormal' on account of increased volume, colour (most often 'white') or malodour. The complaint is associated with considerable self-reported disability, health seeking and associated costs of illness. The standard clinical approach to AVD is syndromic, which is based on the assumption that the complaint is the result of sexual tract infections (STIs), notably Chlamydia trachomatis infection, gonorrhoea or trichomoniasis. Sexual tract infections are potential markers for unsafe sexual behaviour and increased vulnerability to HIV/AIDS, and are associated with adverse health outcomes such as infertility, intrauterine growth retardation, pelvic inflammatory disease, stillbirth and premature labour. Although identification and treatment of STIs are priorities in reproductive health programmes, their diagnosis is hampered by the absence of cheap, simple and accurate diagnostic tests. Thus, the World Health Organization developed the syndromic management guidelines for the treatment of STIs in resource-poor settings. The primary target for the syndromic management of STIs is the complaint of AVD. However, the evidence suggests that the association between the complaint of AVD and the presence of STIs is weak (Patel \& Oomman, 1999). A review of community surveys from South Asia reported that although over half of women have gynaecological complaints, less than half of them have laboratory-confirmed STIs. Studies in clinical settings in South Asia, where one might expect a higher proportion of women with STIs, also show that only about a quarter of women with AVD have a laboratory-confirmed STI. Thus, the current syndromic approach, which assumes AVD to be a marker for STI, is ineffective for the management of both the clinical syndrome and STI. The economic cost of syndromic management per true case of STI is enormous, while the social cost of incorrectly labelling a woman as having an STI is incalculable (Patel \& Oomman, 1999).

\section{The Stree Arogya Shodh}

Why so many women in South Asia complain of AVD was the primary research question of the Stree Arogya Shodh (SAS; 'women's health study' in the Konkani language). This study, funded by the Wellcome Trust, was carried out in the state of Goa on the west coast of India between 2001 and 2004. The SAS was implemented through a partnership between a UK academic institution (the London School of Hygiene and Tropical Medicine), the government of Goa's health services department and Sangath, a Goan community-based nongovernmental organisation pioneering mental health research projects (see http://www.sangath.com). The study was one of the largest population-based cohort studies of women's health in South Asia; it involved the collection of both interview-based 